\title{
Minimizing bottleneck nodes of a substrate in virtual network embedding
}

\author{
Adil Razzaq, Peter Sjödin, Markus Hidell \\ School of ICT, KTH-Royal Institute of Technology \\ Stockholm, Sweden \\ \{arazzaq, psj, mahidell\}@ kth.se
}

\begin{abstract}
Network virtualization has been proposed as a way to increase the flexibility of the Internet. This could enable the provisioning of many desired services in the current architecture and allow effective sharing and use of resources. Providing virtual networks (VNs) means that virtual nodes and links need to be embedded in the underlying shared infrastructure. This embedding process, where VNs with resource demands are mapped onto a substrate with finite resources is a challenging and NP-hard problem. In this paper the focus is on mapping the VNs in such a way that node resources in the substrate are not completely exhausted. To achieve this objective, an approach referred to as bottleneck node reduced mapping is presented. This method is evaluated and compared with an approach, where resource exhaustion is not considered.
\end{abstract}

Keywords- Network Virtualization; Virtual Network Embedding; Resource Management; Scalability

\section{INTRODUCTION}

Network virtualization is an important tool for addressing the Internet ossification [7, 9, 11]. It can be utilized in experimental research facilities $[13,14,15]$ as well as in provision of customized end-to-end services over a shared infrastructure [7, 9]. A number of virtual networks (VNs) can be deployed on top of the physical network (or substrate), depending on the capability of the substrate and the demands of VNs.

The virtual network embedding (VNE) problem is NPhard $[8,17]$ where several constraints need to be satisfied. In order to map a VN onto the substrate, requirements of both its vertices as well as edges should be fulfilled. In addition to this, VNs can arrive at different times, in any order and can be based on any standard network topology (e.g. star, bus, ring or mesh). The substrate network also has a limited amount of resources. Thus, we need to embed or map a $\mathrm{VN}$ with resource constraints onto the substrate network (SN) which has finite resources.

In the past, various efforts have been made to suggest solutions for embedding VNs onto physical networks. By considering that substrate resources are finite, we make "admission control" an integral part of our solution in contrast to previous solutions proposed in $[4,6,12,18]$. Our solution considers that $\mathrm{VN}$ requests are dynamic and are received online, which is contrary to several other solutions $[6,12,18]$. Some previous work points to specific virtual topologies [12], whereas our solution is not limited to any specific topology. Our approach is inspired to some extent by [8] as we use similar notations to denote both virtual and substrate networks. There are solutions with support for path splitting [10] as well as those considering path migration in the physical network
[8]. Path splitting and migration incur cost complexities and are not within the scope of our proposed solution.

An approach of mapping virtual vertices onto any available substrate node which can satisfy their demand was presented in [2]. That method utilizes the substrate's bandwidth resources in an efficient way but does not take into account the possibility of a node becoming a bottleneck after the node mapping, so that no further $\mathrm{VN}$ request can be served by the bottleneck node. Our proposed solution, in addition to mapping vertices onto nodes which can satisfy their demand, also tries to prevent substrate nodes from becoming bottlenecks during the VN mapping.

The main contributions of this paper are to investigate the effect of reducing the bottleneck nodes (referred to as BNRM-Bottleneck Node Reduced Mapping) and its impact on VN embedding, how substrate nodes and links become bottlenecks and get exhausted as well as to analyze how resources are utilized in the process. Definitions for bottleneck and exhausted nodes and links of a substrate are presented in terms of their capability of mapping vertices and edges of future VN requests. A comparison is given between BNRM and the approach presented in [2], which does not consider that a node can become bottleneck at the time of mapping a VN. An evaluation of node exhaustion limit (NEL) values and their impact on different kinds of substrates is also presented. The overall hypothesis behind our solution is that it should be possible to avoid complete exhaustion of node resources and still maintain or improve the VN embedding possibilities. Reducing the number of bottleneck nodes should leave more available nodes for future $\mathrm{VN}$ requests, something which could be beneficial for the VN embedding process.

The rest of the paper is organized as follows. Section II defines the problem while Section III describes our solution. Section IV presents simulation results, whereas Section V ends the paper with a discussion and conclusions.

\section{Network MODEL AND PROBLEM DESCRIPTION}

The proposed solution represents virtual as well as substrate networks as undirected graphs. The substrate network is represented by, $S=\left(N, A^{N}, L, A^{L}\right)$, whereas the network to be mapped i.e. the $\mathrm{VN}$ is shown by $\mathrm{M}=$ $\left(\mathrm{V}, \mathrm{D}^{\mathrm{V}}, \mathrm{E}, \mathrm{D}^{\mathrm{E}}\right)$. Notations for describing the $\mathrm{VN}$ mapping problem are summarized in Table 1.

Throughout this document when a reference is made to a link or node, it means that it belongs to the substrate, while VN's link and node will be termed as edge and vertex respectively. We consider central processing unit (C.P.U.) as a 
resource for nodes and vertices, and bandwidth to be the resource for edges and links.

\section{Table 1: Notations of VNE problem}

\begin{tabular}{|l|l|}
\hline $\mathrm{S}$ & Substrate network \\
$\mathrm{N}$ & Set of nodes belonging to the substrate network \\
$\mathrm{A}^{\mathrm{N}}$ & Attribute associated with substrate nodes \\
$\mathrm{L}$ & Set of links joining two nodes $\left(\mathrm{n}_{\mathrm{i}}, \mathrm{n}_{\mathrm{j}} \in \mathrm{N}\right)$, of the substrate network \\
$\mathrm{A}^{\mathrm{L}}$ & Attribute associated with substrate links \\
$\mathrm{P}_{\mathrm{m}, \mathrm{n}}^{\mathrm{S}}$ & Substrate path from source node $\mathrm{m}$, to destination node $\mathrm{n}$ \\
\hline $\mathrm{M}$ & Virtual network \\
$\mathrm{V}$ & Set of vertices of virtual network \\
$\mathrm{D}^{\mathrm{V}}$ & Demand associated with vertices \\
$\mathrm{E}$ & Set of edges connecting two vertices $\left(\mathrm{v}_{\mathrm{s}}, \mathrm{v}_{\mathrm{t}} \in \mathrm{V}\right)$, of the virtual \\
& network \\
$\mathrm{D}^{\mathrm{E}}$ & Demand associated with edges \\
\hline
\end{tabular}

Fig. 1 shows a substrate network whereas Fig. 2 represents a $\mathrm{VN}$ request. The notation for describing the node and link capacities is similar to the one proposed in [8].

The VN will only be mapped on the substrate if requirements of each of its vertex as well as edge are satisfied. After mapping vertices onto the nodes which satisfy vertex demand, paths need to be calculated for each pair of nodes in the VN. Then the link resources in the path are compared with the edge demand. At this point, if the path satisfies the edge request, then the $\mathrm{VN}$ is completely mapped. After satisfying requests of vertices and edges of a $\mathrm{VN}$, a residual graph $\left(\mathrm{P}_{\text {res }}\right)$ is obtained which contains remaining capacities of nodes and links of the substrate [5].

In the beginning of $\mathrm{VN}$ embedding process, we initialize residual capacities of nodes and links with the actual capacities:

$$
\mathrm{R}^{\mathrm{N}}=\mathrm{A}^{\mathrm{N}}
$$$$
\mathrm{R}^{\mathrm{L}}=\mathrm{A}^{\mathrm{L}}
$$

Therefore, when a node or link is mapping a vertex or an edge for the first time then, its residual capacity is equal to its original capacity $\left(r^{n}=a^{n} \wedge r^{l}=a^{l}\right)$ and the vertex or edge demand $\left(\mathrm{d}^{\mathrm{v}}\right.$ or $\left.\mathrm{d}^{\mathrm{e}}\right)$ is matched with it. After the initial mapping of a vertex or edge is made, the new residual capacity $\left(r^{n}\right)$ of a node $(n \in N)$ is obtained by subtracting vertex demand $\left(d^{v}\right)$ from the node resource, whereas remaining capacity $\left(\mathrm{r}^{\mathrm{l}}\right)$ of a link $(l \in L)$ is found by deducting edge request $\left(\mathrm{d}^{\mathrm{e}}\right)$ from the link resource:

$$
\begin{aligned}
& r^{n} \leftarrow r^{n}-d^{v} \\
& r^{1} \leftarrow r^{1}-d^{e}
\end{aligned}
$$

The resources need to be returned to the substrate if, after mapping the initial vertices or edges of a VN, there comes a point when requirements of a certain edge or vertex cannot be satisfied. This means that in such a scenario the initial or base graph $\left(\mathrm{P}_{\text {base }}\right)$ is regenerated:

$$
\begin{aligned}
& \mathrm{r}^{\mathrm{n}} \leftarrow \mathrm{r}^{\mathrm{n}}+\mathrm{d}^{\mathrm{v}} \\
& \mathrm{r}^{\mathrm{l}} \leftarrow \mathrm{r}^{\mathrm{l}}+\mathrm{d}^{\mathrm{e}}
\end{aligned}
$$

We define the cost of mapping a VN, as the sum of overall substrate resources assigned to its vertices and edges, in the same way, as previously presented in [10]. Our cost function is similar to the one given in [10]:

$$
\mathrm{C}(\mathrm{M})=\Sigma_{\mathrm{e} \in \mathrm{E}} \Sigma_{\mathrm{l} \in \mathrm{L}}\left(\mathrm{b}_{\mathrm{e}}^{\mathrm{l}}\right)+\Sigma_{\mathrm{v} \in \mathrm{V}}\left(\mathrm{d}^{\mathrm{v}}\right)
$$

A vertex will only be mapped on a single node whereas the edge can be mapped on a substrate path $\left(\mathrm{P}_{\mathrm{m}, \mathrm{n}}^{\mathrm{S}}\right)$ containing one or more than one links. The term $\left(b_{\mathrm{e}}^{1}\right)$ in (4) indicates bandwidth allocated to an edge $(e \in E)$ from a substrate link $(l \in \mathrm{L})$.
The proposed solution can map both topology specific VNs (e.g., VN represented in Fig. 2 is based on ring topology) as well as randomly generated $\mathrm{VNs}$.
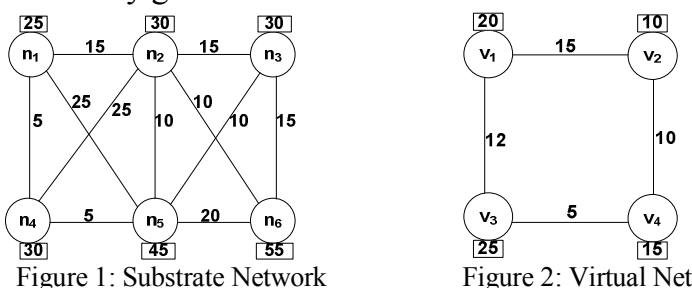

Figure 2: Virtual Network

\section{VIRTUAL NETWORK EMBEDDING}

The VN mapping process starts by assigning vertices to nodes, then proceeds on to find edge disjoint $\mathrm{k}$-shortest paths [3] between each pair of nodes and finishes by mapping edges onto the paths that satisfy their demand.

The first step of the proposed solution starts by finding candidate nodes of the substrate, which can map vertices by satisfying their demands. In this phase, each vertex, $(\mathrm{V} \in$ $\left.\mathrm{V} \wedge \mathrm{d}^{\mathrm{V}} \in \mathrm{D}^{\mathrm{V}}\right)$, has to be mapped to a different node, $(\mathrm{n} \in$ $\mathrm{N} \wedge \mathrm{a}^{\mathrm{n}} \in \mathrm{A}^{\mathrm{N}}$ ). The approach of mapping vertices on the first available substrate node which can satisfy their demand (by only evaluating if, $\mathrm{r}^{\mathrm{n}} \geq \mathrm{d}^{\mathrm{v}}$ ) was presented in [2]. In this paper we consider that method as a baseline and term it as BLA (baseline approach). In the next step, we add an extension to the BLA by adding the node exhaustion limit (nel) feature, call it BNRM (bottleneck node reduced mapping), and make a comparison between the two approaches. The main difference between BNRM and BLA is that, the latter does not consider the possibility of a node becoming a bottleneck at the time of mapping a vertex.

Node exhaustion limit (nel) is a value which is used to make sure that a node does not become bottleneck. The vertex is only mapped on the node if, after mapping, the node has resources equal to or greater than nel. In the start of $\mathrm{VN}$ embedding process, nel is set to a comparatively higher value, since the substrate has all the resources intact in the beginning. The nel value is decreased after a certain number of requests have arrived, since, after mapping the initial VNs, the substrate also has less number of available resources. Another important thing behind this concept is that in case a substrate has more resources on its nodes and demands for vertices of VNs is less, then, instead of selecting same set of nodes each time, a different set of nodes could be selected. Since node mapping affects link mapping, so, if same nodes keep on getting selected repeatedly then resources on their links can become exhausted. The nel $\mathrm{l}_{\mathrm{t}}$ in (5), defines the time at which a $\mathrm{VN}$ arrives and is compared with a certain nel value. The vertex mapping function for BNRM can be given as:

\section{Subject to:}

$$
\mathrm{V}^{\mathrm{M}}:\left(\mathrm{V}, \mathrm{D}^{\mathrm{V}}\right) \rightarrow\left(\mathrm{N}, \mathrm{A}^{\mathrm{N}}\right)
$$

$$
\left(\left(\mathrm{r}^{\mathrm{n}}-\mathrm{d}^{\mathrm{v}}\right) \geq \mathrm{nel}_{\mathrm{t}}\right)
$$

The vertex mapping function in (5), starts by checking all the vertices $(\mathrm{V})$ and first selects the one which demands most processor resources $\left(\max \left(\mathrm{D}^{\mathrm{V}}\right)\right)$. The benefit of doing this is that if, the substrate cannot satisfy the demand of this vertex then the mapping process stops here for this $\mathrm{VN}$ and the requirements of the remaining vertices don't need to be checked, which saves amount of computations according to the number of vertices in a VN. In this way, if demand of the 
first vertex is satisfied then the process is repeated for all the remaining vertices moving on from vertex demanding most to the least resources.

The vertex mapping algorithm is defined below:

Algorithm 1: Vertex mapping

1: Take a $\mathrm{VN}$ request.

2: Find a unique substrate node, for every vertex, having sufficient resources to satisfy the CPU demands, (according to the selected vertex mapping function, BNRM or BLA), start from the vertex demanding the most resources.

3: If all the vertices can be mapped at this stage, then, generate residual node capacities according to (2a), else, GOTO 5.

4: Call "edge mapping algorithm".

5: If this was the last request, then, stop, else, GOTO 1.

The VN requests are satisfied by using the first come first served (FCFS) approach and the process begins by assigning vertices to the substrate nodes. The edge mapping algorithm is called only if, the vertex requests can be satisfied at this stage.

The next step is to map an edge $\left(e \in E \wedge d^{e} \in D^{E}\right)$ on a substrate path $\left(\mathrm{P}_{\mathrm{m}, \mathrm{n}}^{\mathrm{S}}\right)$ containing one or more than one links. In the proposed solution, first $\mathrm{k}$-shortest paths are found for each edge. The next step is to calculate resources on each path. To achieve this objective, we take link with minimum resources in the path $\left(\min \left(\mathrm{P}_{\mathrm{m}, \mathrm{n}}^{\mathrm{S}}\right)\right)$ and match edge demand with it. If this link can satisfy edge request, then the remaining links will surely be able to do that.

The approach of mapping an edge on shortest of the kshortest paths, which can satisfy its demand, termed as shortest path mapping (SPM), was presented in [2, 8]. The edge mapping function for the SPM can be given as:

Subject to

$$
E^{M}:\left(E, D^{E}\right) \rightarrow P_{m, n}^{S}
$$

$$
\left(\mathrm{d}^{\mathrm{e}} \leq\left(\min \left(\mathrm{P}_{\mathrm{m}, \mathrm{n}}^{\mathrm{S}}\right)\right)\right)
$$

The edge mapping algorithm is defined below:

Algorithm 2: Edge mapping

1: Take the request which has successfully passed the vertex mapping stage.

2: for (each_edge_in_the_request) do

2.1: Search the k-shortest paths between the pair of vertices connected by the edge (mapped on nodes by the vertex mapping algorithm).

2.2: Select the path which satisfies the edge demand (according to the edge mapping function, SPM).

2.3: If edge request is not satisfied, then, GOTO 3, else, generate residual link capacities of the selected path according to (2b) and GOTO 4.

2.4: end for

3: If this was the first edge of VN, then, return node resources to the substrate according to (3a), else, return both the link and node resources to the substrate (as defined in $3 a$ and $3 b$ ).

4: If this was the last request, then, stop, else, call "vertex mapping algorithm".
Edge mapping phase of the solution assigns all edges to the substrate paths and is executed number of times the total edges in a VN. It starts by finding out edge disjoint k-shortest paths for each edge of the VN. In the next step, resources on each of these paths are calculated and the path having sufficient resources, satisfying the edge demand, is selected. At this point, if there are sufficient path resources to satisfy the request of all the edges then the VN is completely mapped (request satisfied).

\section{EXPERIMENTAL SETUP AND EVALUATION}

This section is divided into two parts; first describes experimental setup while second presents the evaluation results. The proposed solution has been implemented using Matlab®.

\section{A. Experimental Setup}

The substrate networks have been generated using the BRITE tool [1] whereas, virtual networks have been created using Matlab®.

\section{1) Substrate Networks}

The proposed solution has been tested on different substrate networks. Each of these networks consists of 100 nodes and about 500 links, and node resource (C.P.U.) as well as link resource (bandwidth) is assigned different values from 1 to 100 units $[2,5,8]$. The size of substrates can be compared with that of a medium sized ISP [8]. The substrates have been assigned different number of resources to see the effect of nel values, on substrates with same size but different amount of resources. The three substrates used are:

$\mathrm{S}_{1}$ : Node and link resources are randomly chosen from 1 to 100.

$\mathrm{S}_{2}$ : Node and link resources are randomly chosen from 10 to 100 .

$\mathrm{S}_{3}$ : Node and link resources are randomly chosen from 20 to 100 .

\section{2) Virtual Networks}

Two different sets of VNs have been mapped onto substrate networks; each set differs from the other, by number of edges.

Set 1: The number of vertices of a $\mathrm{VN}$ is randomly chosen between 2 and 10 and the vertices are randomly connected with the probability 0.3 .

Set 2: The number of vertices of a $\mathrm{VN}$ is randomly chosen between 2 and 10 and the vertices are randomly connected with the probability 0.5 .

The set- 2 is similar to the setup presented in $[2,5,8,12]$ while set-1 resembles with the one given in [2]. The vertex and edge resources in both sets are randomly chosen from 1 to 5. The reason for selecting low values for vertices is to evaluate the concept of nel as described in section-III.

\section{B. Evaluation}

The main focus of evaluation is to study the effect of minimizing bottleneck nodes of a substrate, their impact on mapping $\mathrm{VN}$ requests, the way resources are utilized, nodes and links become bottleneck and get exhausted.

The VNs belonging to set-1 put less demand on substrate's paths whereas, VNs from set-2 are well connected. Therefore, it is interesting to look at the comparison from the point of view of two sets of VNs: 
(i) VN set-1:

(a) VN Mapping $\left(\operatorname{Map}\left(M_{n}\right)\right)$

Figure 3 to 5 show mapping of $\mathrm{VNs}$ and cost incurred in the process. In some cases, BLA approach maps more VNs than BNRM for initial VNs in a set (Fig. 3). However, the benefit of BNRM can be seen as number of requests grows, as it can map more requests in later stages of $\mathrm{VN}$ embedding process as compared to BLA (Fig. 3).

We used same set of nel values for all the substrates $\left(\mathrm{S}_{1}, \mathrm{~S}_{2}\right.$ and $\mathrm{S}_{3}$ ). The BNRM approach maps more overall VNs than BLA for $S_{1}$, and almost same for $S_{3}$ (Figure 3 and 5). However, the results are different for $\mathrm{S}_{2}$ (Fig. 4). This lead to the evaluation of selecting different set of nel values for different substrates, which is analyzed and presented later.

The two approaches were further analyzed in Table 2 and 3 for the rest of evaluation criteria, which represent, analysis of VN-set1 on $\mathrm{S}_{1}$. The main focus of this paper is to introduce BNRM approach to minimize bottleneck nodes of the substrate, but node mapping affects link mapping and therefore, it is interesting to study the state of substrate links as well.

(b) Resource Utilization $\left(U\left(S_{r}\right)\right.$ )

The resource utilization should be viewed based on the following factors:

(i) When same number of VNs are mapped by the two methods at a particular instant of time.

(ii) The overall VNs mapped by using a certain method.

The two approaches map almost same number of VNs when initial $100 \mathrm{VN}$ requests arrive (Fig. 3). When same number of VNs gets mapped, the BNRM utilizes more substrate resources (Table 2 and 3). The average cost of mapping a $\mathrm{VN}, \mathrm{C}\left(\mathrm{M}_{\mathrm{avg}}\right)$, at this point for $\mathrm{BNRM}$ is 40.16 units of substrate resources, whereas $C\left(M_{a v g}\right)$ for BLA is 34.29 units of substrate resources.

In addition to this, the BNRM utilizes $6.94 \%$ more overall resources as well (Table 2 and 3) since; it can overall map $7.25 \%$ more VNs as compared to the BLA (Table 2 and 3 ).

\section{(c) Bottleneck Nodes $(B(N))$}

The idea to minimize the use of substrate resources from bottleneck nodes and links was presented in [8], while, the concept of bottleneck links was also mentioned in [16]. Nodes and links having lack of residual capacities to map vertices/edges and hence resulting in rejection of a $\mathrm{VN}$ request were termed as bottlenecks in [17]. One of the contributions of this paper is that, we give definitions for bottleneck nodes and links of a substrate in terms of their capability of mapping vertices and edges of VNs due to arrive in future in the mapping process.

We define a node as a bottleneck if, its residual capacity is less than a certain value $\left(\mathrm{r}^{\mathrm{n}}<\mathrm{val}_{\mathrm{n}}\right)$. The value chosen for nodes in Table 2 to 5 , is $10\left(\operatorname{val}_{n}=10\right)$. A node does not become bottleneck if it is able to map at least two vertices. Since, for both sets of VNs, the maximum capacity of a vertex can be 5 therefore; the value chosen for $\operatorname{val}_{n}$ is 10 .

The nodes start becoming bottleneck from VN interval-1(VNs $1-50$ ) when, BLA is used for mapping (Table 2 ). The readings in Table 2 and 3 are shown from substrate $S_{1}$, which contains resources in the range 1 to 100 . Therefore, according to

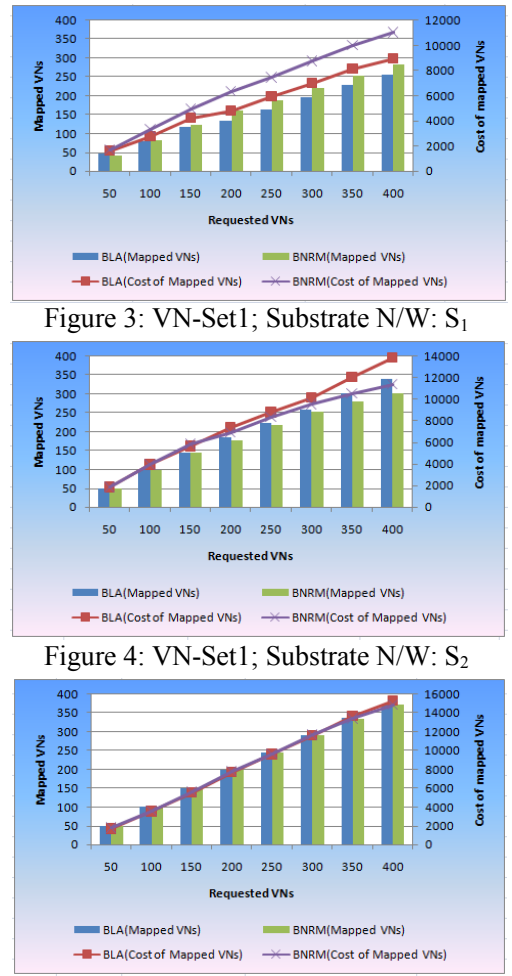

Figure 5: VN-Set1; Substrate N/W: $\mathrm{S}_{3}$

definition, $11 \%$ nodes are already bottlenecks before the start of $\mathrm{VN}$ embedding process (nodes contain values which are less than 10). When mapping of VNs starts and vertex mapping approach is BNRM then there is no further bottleneck till the fifth interval ( $250 \mathrm{VNs}$, Table 3$)$. This ensures that the substrate has more node resources in place, and therefore, $10 \%$ more $\mathrm{VNs}$ are mapped in the last interval (VNs 351-400) using BNRM (Table 2 and 3).

(d) Exhausted Nodes $(E(N))$

An exhausted node is a bottleneck node, whose resources get completely utilized $\left(\mathrm{r}^{\mathrm{n}}=0\right)$. Table 3 shows that, only $34 \%$ of the node resources get exhausted when BNRM is used, as compared to $72 \%$ when vertex mapping approach is BLA (Table 2). This ensures that the substrate still has 38\% more usable nodes for the future VN requests (Table 2 and 3 ).

(e) Bottleneck Links $(B(L))$

Similar to the definition of a bottleneck node, a link is defined as a bottleneck if, its residual capacity $\left(\mathrm{r}^{\mathrm{l}}\right)$ is less than a certain value $\left(\mathrm{r}^{\mathrm{l}}<\mathrm{val}_{1}\right)$. The value chosen for links in Table 2 to 5 , is $10\left(\right.$ val $\left._{l}=10\right)$. A link does not become bottleneck if, it is able to map at least two edges.

When approximately same number of VNs gets mapped (up to $100 \mathrm{VNs}$ ), BNRM makes $2.4 \%$ more bottleneck links (Table 2 and 3). Overall as well, BNRM makes 5.6\% more links as bottlenecks as compared to the BLA (Table 2 and 3).

(f) Exhausted Links (E(L))

A bottleneck link becomes exhausted, when all its resources are utilized $\left(\mathrm{r}^{1}=0\right)$. When approximately same number of VNs gets mapped (up to $100 \mathrm{VNs}$ ), the BNRM exhausts $1.4 \%$ more links (Table 2 and 3). Overall as well, $6.4 \%$ more links become exhausted when the method used is BNRM (Table 2 and 3). 
Table 2: BLA Resource Utilization (VN-Set1; Substrate N/W: $\mathrm{S}_{1}$ )

\begin{tabular}{|c|c|c|c|c|c|c|c|}
\hline Req $\left(\mathrm{M}_{\mathrm{n}}\right)$ & $\mathrm{Map}\left(\mathrm{M}_{\mathrm{n}}\right)$ & $\mathrm{C}\left(\mathrm{M}_{\mathrm{n}}\right)$ & $\mathrm{U}\left(\mathrm{S}_{\mathrm{r}}\right)$ & $\mathrm{B}(\mathrm{N})$ & $\mathrm{E}(\mathrm{N})$ & $\mathrm{B}(\mathrm{L})$ & $\mathrm{E}(\mathrm{L})$ \\
\hline $1-50$ & $92 \%$ & 1627 & $5.44 \%$ & $24 \%$ & $12 \%$ & $13.6 \%$ & $4 \%$ \\
\hline $51-100$ & $68 \%$ & 1116 & $3.73 \%$ & $7 \%$ & $12 \%$ & $3.2 \%$ & $3.2 \%$ \\
\hline $101-50$ & $76 \%$ & 1480 & $4.95 \%$ & $10 \%$ & $9 \%$ & $4.2 \%$ & $3.4 \%$ \\
\hline $151-200$ & $32 \%$ & 593 & $1.98 \%$ & $4 \%$ & $4 \%$ & $1.8 \%$ & $1.6 \%$ \\
\hline $201-250$ & $58 \%$ & 1125 & $3.76 \%$ & $10 \%$ & $10 \%$ & $3.8 \%$ & $3.4 \%$ \\
\hline $251-300$ & $64 \%$ & 1065 & $3.56 \%$ & $9 \%$ & $10 \%$ & $1.2 \%$ & $3.8 \%$ \\
\hline $301-350$ & $68 \%$ & 1102 & $3.69 \%$ & $7 \%$ & $9 \%$ & $2.2 \%$ & $1.8 \%$ \\
\hline $351-400$ & $52 \%$ & 830 & $2.78 \%$ & $6 \%$ & $6 \%$ & $2.6 \%$ & $2.8 \%$ \\
\hline $1-400$ & $63.75 \%$ & 8938 & $29.9 \%$ & $77 \%$ & $72 \%$ & $32.6 \%$ & $24 \%$ \\
\hline
\end{tabular}

Table 3: BNRM Resource Utilization (VN-Set1; Substrate N/W: $\mathrm{S}_{1}$ )

\begin{tabular}{|l|c|c|c|c|c|c|c|}
\hline Req $\left(\mathrm{M}_{\mathrm{n}}\right)$ & Map $\left(\mathrm{M}_{\mathrm{n}}\right)$ & $\mathrm{C}\left(\mathrm{M}_{\mathrm{n}}\right)$ & $\mathrm{U}\left(\mathrm{S}_{\mathrm{r}}\right)$ & $\begin{array}{c}\mathrm{B} \\
(\mathrm{N})\end{array}$ & $\begin{array}{c}\mathrm{E} \\
(\mathrm{N})\end{array}$ & $\mathrm{B}(\mathrm{L})$ & $\mathrm{E}(\mathrm{L})$ \\
\hline $1-50$ & $84 \%$ & 1690 & $5.65 \%$ & $11 \%$ & 0 & $14 \%$ & $5.2 \%$ \\
\hline $51-100$ & $82 \%$ & 1643 & $5.50 \%$ & 0 & 0 & $5.2 \%$ & $3.4 \%$ \\
\hline $101-150$ & $82 \%$ & 1613 & $5.40 \%$ & 0 & 0 & $4.4 \%$ & $3.6 \%$ \\
\hline $151-200$ & $72 \%$ & 1396 & $4.67 \%$ & 0 & 0 & $2.4 \%$ & $4.2 \%$ \\
\hline $201-250$ & $54 \%$ & 1091 & $3.65 \%$ & 0 & 0 & $3 \%$ & $3 \%$ \\
\hline $251-300$ & $64 \%$ & 1309 & $4.38 \%$ & $67 \%$ & 0 & $3.6 \%$ & $4.8 \%$ \\
\hline $301-350$ & $68 \%$ & 1250 & $4.18 \%$ & 0 & 0 & $3.6 \%$ & $3.2 \%$ \\
\hline $351-400$ & $62 \%$ & 1021 & $3.42 \%$ & $8 \%$ & $34 \%$ & $2 \%$ & $3 \%$ \\
\hline $1-400$ & $71 \%$ & 11013 & $\begin{array}{c}36.84 \\
\%\end{array}$ & $86 \%$ & $34 \%$ & $\begin{array}{c}38.2 \\
\%\end{array}$ & $\begin{array}{c}30.4 \\
\%\end{array}$ \\
\hline
\end{tabular}

(ii) VN set-2:

(a) VN Mapping $\left(\operatorname{Map}\left(M_{n}\right)\right)$

The VNs in set-2 are well connected. Therefore, if nodes get mapped at a distance then, the chances of denial of a VN request may also get increased. When overall mapped VNs are considered the BNRM comes close to BLA (Figure 6 to 8) but falls just short. However, the advantage of reserving resources for future requests is evident here as well (Table 5). A comparison of Table 4 and 5 shows that for the last interval (VNs 351-400), the BNRM can still map 20\% more VNs as compared to the BLA (Table 4 and 5). However, more VNs get rejected initially and overall BLA maps $1 \%$ more VNs (Table 4 and 5).

The two approaches were further analyzed in Table 4 and 5 for rest of the evaluation criteria, which represents, analysis of VN-set2 on substrate $\mathrm{S}_{1}$.

\section{(b) Resource Utilization $\left(U\left(S_{r}\right)\right)$}

The two approaches don't map same number of VNs from the initial set on substrate $S_{1}$. Therefore, a cost comparison for this substrate cannot be made. However, on substrate $\mathrm{S}_{3}$, both methods map almost the same number of VNs when initial 50 requests arrive (Fig. 8). At this point the BLA uses less substrate resources than BNRM (Fig. 8).

(c) Bottleneck Nodes $(B(N))$

When BNRM is used, there are no bottleneck nodes till the fifth interval (similar explanation like given for $\mathrm{VN}$-set1). This ensures that the substrate has more node resources in place (Table 5), and therefore, $20 \%$ more VNs get mapped in the last interval using BNRM (Table 4 and 5).

(d) Exhausted Nodes ( $E(N)$ )

The substrate, at the end of VN mapping process, still has $28 \%$ more usable nodes using BNRM as compared to BLA, as shown by Table 4 and 5 .

The results for VN set-2 are similar to VN set-1 in terms of bottleneck and exhausted links (Table 4 and 5).

\section{Discussion:}

The BNRM approach produces more usable substrate nodes for future VN requests. A comparison of Table 2 and 3

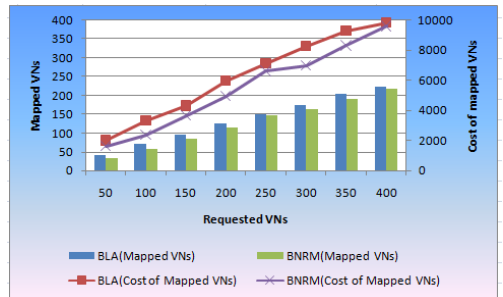

Figure 6: VN-Set2; Substrate N/W: $\mathrm{S}_{1}$

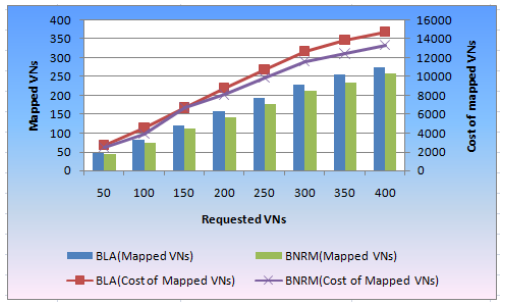

Figure 7: VN-Set2; Substrate N/W: $\mathrm{S}_{2}$

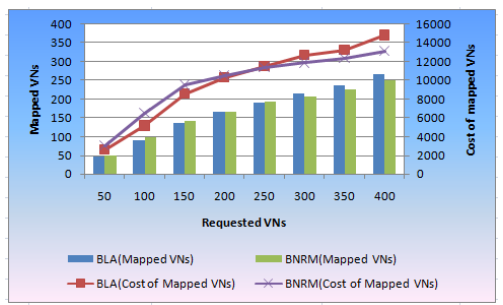

Figure 8: VN-Set2; Substrate N/W: $\mathrm{S}_{3}$

Table 4: BLA Resource Utilization (VN-Set2; Substrate N/W: $\mathrm{S}_{1}$ )

\begin{tabular}{|c|c|c|c|c|c|c|c|}
\hline $\operatorname{Req}\left(\mathrm{M}_{\mathrm{n}}\right)$ & Map $\left(\mathrm{M}_{\mathrm{n}}\right)$ & $\mathrm{C}\left(\mathrm{M}_{\mathrm{n}}\right)$ & $\mathrm{U}\left(\mathrm{S}_{\mathrm{r}}\right)$ & $\begin{array}{c}\mathrm{B} \\
(\mathrm{N})\end{array}$ & $\begin{array}{c}\mathrm{E} \\
(\mathrm{N})\end{array}$ & $\mathrm{B}(\mathrm{L})$ & $\mathrm{E}(\mathrm{L})$ \\
\hline $1-50$ & $80 \%$ & 1994 & $6.67 \%$ & $22 \%$ & $10 \%$ & $\begin{array}{c}15.2 \\
\%\end{array}$ & $5 \%$ \\
\hline $51-100$ & $62 \%$ & 1319 & $4.41 \%$ & $6 \%$ & $9 \%$ & $5 \%$ & $3.8 \%$ \\
\hline $101-150$ & $48 \%$ & 990 & $3.31 \%$ & $7 \%$ & $8 \%$ & $2.4 \%$ & $4.4 \%$ \\
\hline $151-200$ & $60 \%$ & 1673 & $5.60 \%$ & $6 \%$ & $6 \%$ & $7 \%$ & $5.6 \%$ \\
\hline $201-250$ & $50 \%$ & 1142 & $3.82 \%$ & $5 \%$ & $5 \%$ & $2.2 \%$ & $4.2 \%$ \\
\hline $251-300$ & $50 \%$ & 1146 & $3.83 \%$ & $9 \%$ & $9 \%$ & $2 \%$ & $2.4 \%$ \\
\hline $301-350$ & $58 \%$ & 1000 & $3.35 \%$ & $9 \%$ & $9 \%$ & $1.8 \%$ & $2.2 \%$ \\
\hline $351-400$ & $36 \%$ & 559 & $1.87 \%$ & $3 \%$ & $3 \%$ & $0.8 \%$ & $1 \%$ \\
\hline $1-400$ & $55.5 \%$ & 9823 & $\begin{array}{c}32.86 \\
\%\end{array}$ & $67 \%$ & $59 \%$ & $\begin{array}{c}56.4 \\
\%\end{array}$ & $\begin{array}{c}28.6 \\
\%\end{array}$ \\
\hline
\end{tabular}

Table 5: BNRM Resource Utilization (VN-Set2; Substrate N/W: $\mathrm{S}_{1}$ )

\begin{tabular}{|c|c|c|c|c|c|c|c|}
\hline Req $\left(\mathrm{M}_{\mathrm{n}}\right)$ & Map $\left(\mathrm{M}_{\mathrm{n}}\right)$ & $\mathrm{C}\left(\mathrm{M}_{\mathrm{n}}\right)$ & $\mathrm{U}\left(\mathrm{S}_{\mathrm{r}}\right)$ & $\begin{array}{c}\mathrm{B} \\
(\mathrm{N})\end{array}$ & $\begin{array}{c}\mathrm{E} \\
(\mathrm{N})\end{array}$ & $\mathrm{B}(\mathrm{L})$ & $\mathrm{E}(\mathrm{L})$ \\
\hline $1-50$ & $68 \%$ & 1603 & $5.36 \%$ & $11 \%$ & 0 & $\begin{array}{c}14.8 \\
\%\end{array}$ & $\begin{array}{c}4.8 \\
\%\end{array}$ \\
\hline $51-100$ & $46 \%$ & 744 & $2.49 \%$ & 0 & 0 & $1.4 \%$ & $2 \%$ \\
\hline $101-150$ & $58 \%$ & 1313 & $4.39 \%$ & 0 & 0 & $4.8 \%$ & $4 \%$ \\
\hline $151-200$ & $58 \%$ & 1263 & $4.22 \%$ & 0 & 0 & $5 \%$ & $\begin{array}{c}4.4 \\
\%\end{array}$ \\
\hline $201-250$ & $62 \%$ & 1692 & $5.66 \%$ & 0 & 0 & $3.2 \%$ & $\begin{array}{c}4.6 \\
\%\end{array}$ \\
\hline $251-300$ & $36 \%$ & 376 & $1.26 \%$ & $34 \%$ & 0 & $1.4 \%$ & $\begin{array}{c}0.2 \\
\%\end{array}$ \\
\hline $301-350$ & $52 \%$ & 1314 & $4.40 \%$ & $16 \%$ & 0 & $1.4 \%$ & $\begin{array}{c}4.8 \\
\%\end{array}$ \\
\hline $351-400$ & $56 \%$ & 1278 & $4.27 \%$ & $6 \%$ & $31 \%$ & $4 \%$ & $\begin{array}{c}2.2 \\
\%\end{array}$ \\
\hline $1-400$ & $54.5 \%$ & 9583 & $\begin{array}{c}32.06 \\
\%\end{array}$ & $67 \%$ & $31 \%$ & $36 \%$ & $27 \%$ \\
\hline
\end{tabular}

indicates that $38 \%$ more usable nodes are produced by BNRM method when compared with BLA. But, a comparison of Table 2 and 3 also shows that $5.6 \%$ more links become bottleneck when approach used is BNRM. However, in spite 
of making more bottleneck links and utilization of more substrate resources, BNRM still maps $7.25 \%$ more overall VNs as compared to BLA for less connected VNs (Table 2 and 3). We evaluated three different $\mathrm{VN}$ sets of this type and found consistent results (not shown due to space limitations).

The results are similar in terms of producing more usable nodes when two approaches are compared for a VN set comprising of VNs which put more demand on substrate links (Section IV, VN set-2). In this case, BNRM still produces $28 \%$ more usable nodes as compared to BLA (Table 4 and 5). However, by using BNRM there is a possibility of mapping vertices on nodes which may be at a distance, which also increases possibility of rejection of a $\mathrm{VN}$ request. Therefore, in spite of having more usable nodes for the future $\mathrm{VN}$ requests, and mapping more $\mathrm{VN}$ requests in later stages of mapping process, more requests get rejected initially and overall a few less VNs get mapped (Table 4 and 5).

Node Exhaustion Limit (nel):

The results of Fig. 4 indicated that a further evaluation of nel values was required. The results of new evaluations are not shown (due to space limitations) and only the conclusions drawn are presented. The following three sets of nel values were used:

Set A: Starting value of nel is $35 \%$ of maximum node resource. There is a decrease of 5\% after every interval (after every $40 \mathrm{VNs}$ ) and values for last three intervals are 1, 1 and 0 .

Set B: Starting value of nel is $40 \%$ of maximum node resource. There is a decrease of 5\% after every interval and the values for the last two intervals are 1 and 0 .

Set C: Starting value of nel is $50 \%$ of maximum node resource. There is a decrease of $10 \%$ for first two intervals, and a reduction of 5\% for next five intervals, whereas values for last two intervals are 1 and 0 .

The criteria chosen for best value set for a substrate is based on the following factors:

(i) The least number of VNs rejected in the beginning of the embedding process.

(ii) The overall VNs mapped at the end.

We conclude that nel value set should be based on amount of resources in the substrate. A set of high values for a substrate containing fewer resources can map vertices on nodes which are far from each other, which can result in longer paths, utilize more bandwidth resources and also increases possibility of rejection of a VN request (e.g., use of set-C on substrate $S_{1}$ ). On the other hand, a set of small values, for a substrate containing higher number of resources can result in selecting same set of nodes repeatedly which can exhaust link resources early (e.g., use of set-A on substrate $\mathrm{S}_{2}$ ). The use of a set of very high nel values should be avoided unless the substrate has similar number of resources as nel value set (e.g., use of set-C on substrate $\mathrm{S}_{3}$ ). A moderately selected set of nel values can on average give sufficient VN mappings and can also result in rejection of fewer $\mathrm{VN}$ requests (e.g., use of set-B on substrates $\mathrm{S}_{1}, \mathrm{~S}_{2}$ and $\mathrm{S}_{3}$ ).

\section{CONCLUSION AND FUTURE WORK}

We have investigated an approach to virtual network embedding where we attempt to assign virtual networks into a substrate in a way that avoids exhausting node resources completely. The idea is that with this approach, some resources are left for the benefit of future embedding requests, which could improve the overall embedding capability.

We investigate our approach (referred to as BNRMBottleneck Node Reduced Mapping) and compare it to an alternative approach (BLA) that does not take node resource exhaustion into consideration. The number of networks that can be embedded is investigated for varying distributions of virtual network requests and for different substrate topologies. The results indicate that BNRM is favorable for more sparsely connected networks, while for denser networks, BLA shows similar or slightly better performance. Therefore, one possible extension of this work could be to investigate how the two approaches could be combined in order to maximize the number of virtual networks that are mapped, while still avoiding bottleneck nodes.

\section{REFERENCES}

[1] A. Medina, A. Lakhina, I. Matta, and J. Byers, "BRITE: Universal topology generation from a user's perspective", Boston University, BUCS-TR- 2001-003, 2001.

[2] A. Razzaq and M. S. Rathore, "An Approach towards Resource Efficient Virtual Network Embedding," internet, pp. 68-73, 2010 2nd International Conference on Evolving Internet, 2010.

[3] D. Eppstein, "Finding the k shortest paths", In Proc. IEEE Symposium on Foundations of Computer Science, pp. 154-165, 1994.

[4] J. Fan and M. Ammar, "Dynamic topology configuration in service overlay networks: A study of reconfiguration policies", In Proc. IEEE INFOCOM, 2006.

[5] J. Lischka and H. Karl, "A virtual network mapping algorithm based on subgraph isomorphism detection", ACM SIGCOMM, 2009.

[6] J. Lu and J. Turner, "Efficient mapping of virtual networks onto a shared substrate", Washington University, Technical Report WUCSE-2006$35,2006$.

[7] J. S. Turner and D. E. Taylor, "Diversifying the Internet", In Proc. IEEE GLOBECOM, pp. 755-760, 2005.

[8] M. Yu, Y. Yi, J. Rexford, and M. Chiang, "Rethinking virtual network embedding: Substrate support for path splitting and migration," ACM SIGCOMM Computer Communication Review, vol. 38, no. 2, pp. 1729, 2008.

[9] N. Feamster, L. Gao, and J. Rexford, "How to lease the Internet in your spare time", SIGCOMM Computer Communication Reiew, vol. 37, no. 1, pp. 61-64, 2007.

[10] N. M. M. K. Chowdhury, M. R. Rahman, and R. Boutaba, "Virtual network embedding with coordinated node and link mapping", IEEE INFOCOM, pp. 783-791, 2009.

[11] T. Anderson, L. Peterson, S. Shenker, and J. Turner, "Overcoming the Internet impasse through virtualization", IEEE Computer Magazine, vol. 38 , no. 4, pp. 34-41, 2005.

[12] Y. Zhu and M. Ammar, "Algorithms for assigning substrate network resources to virtual network components", In Proc. IEEE INFOCOM, pp. $1-12,2006$.

[13] A. Bavier, N. Feamster, M. Huang, L. Peterson, and J. Rexford, "In VINI Veritas: Realistic and controlled network experimentation. In Proc. ACM SIGCOMM, pp. 3-14, 2006.

[14] GENI. http://www.geni.net/.

[15] Planetlab. http://www.planet-lab.org/.

[16] Y. Zhu, "Routing, resource allocation and network design for overlay networks", PhD. thesis, College of computing, Georgia Institute of Technology, 2006.

[17] N. F. Butt, M. Chowdhury, and R. Boutaba, "Topology-Awareness and Reoptimization Mechanism for Virtual Network Embedding", In Proceedings of the 9th IFIP NETWORKING Conference, pp. 27-39, 2010.

[18] I. Houidi, W. Louati, and D. Zeghlache, "A Distributed Virtual Network Mapping Algorithm", In Proceedings of the 2008 IEEE International Conference on Communications (ICC), pp. 5634-5640, 2008. 